\title{
Master of cardiothoracic surgery: transcaval transcatheter aortic valve replacement
}

\author{
Isida Byku ${ }^{1}$, Norihiko Kamioka ${ }^{1}$, Adam B. Greenbaum ${ }^{1}$, Patrick Gleason ${ }^{1}$, Vasilis C. Babaliaros ${ }^{1}$, \\ Kendra J. Grubb ${ }^{2}$ \\ ${ }^{1}$ Structural Heart and Valve Center, Division of Cardiology, Emory University School of Medicine, Atlanta, GA, USA; ${ }^{2}$ Structural Heart and Valve \\ Center, Division of Cardiothoracic Surgery, Emory University School of Medicine, Atlanta, GA, USA \\ Correspondence to: Kendra J. Grubb, MD, MHA, FACC. Surgical Director, Structural Heart and Valve Center Emory University, 550 Peachtree St. \\ NE, Davis-Fischer $4^{\text {th }}$ Floor, Suite 4307, Atlanta, GA 30308, USA. Email: kjgrubb@emory.edu.
}

Submitted Apr 22, 2020. Accepted for publication Jun 02, 2020.

doi: 10.21037/acs-2020-av-17

View this article at: http://dx.doi.org/10.21037/acs-2020-av-17

\section{Clinical vignette}

A 70-year-old veteran with worsening shortness of breath and exertional angina presented for treatment of severe symptomatic aortic stenosis (AS). Relevant medical history included coronary artery disease with coronary artery bypass grafting, chronic kidney disease, hypertension, diabetes mellitus, and prior cerebral vascular accident with residual right sided weakness. Considering the patient's comorbidities and Society of Thoracic Surgery score of $5.152 \%$, the patient and heart team decided to proceed with transcatheter aortic valve replacement (TAVR).

Echocardiography showed heavily calcified leaflets, aortic valve area (AVA) $0.53 \mathrm{~cm}^{2}$ and mean gradient (MG) $42.0 \mathrm{mmHg}$; with preserved left ventricular function. Cardiac gated computed tomography showed aortic annular perimeter of $76.1 \mathrm{~mm}$ and severely diseased bilateral iliofemoral vessels. There was an appropriate window for transcaval TAVR (TC-TAVR).

\section{Surgical technique}

\section{Preparation}

In the hybrid suite, the patient was intubated due to an inability to lie flat. He was prepped and draped for a standard transfemoral-TAVR (TF-TAVR) with both groins and right wrist exposed.

\section{Exposure/access}

Access was obtained under ultrasound and fluoroscopy guidance with placement of sheaths: $8 \mathrm{~F}$ right common femoral vein (RCFV), $7 \mathrm{~F}$ right common femoral artery (RCFA), 7F left common femoral vein (LCFV), and 5/6F right radial artery. The RCFV site was pre-closed with double Perclose ${ }^{\mathrm{TM}}$ technique. The patient was heparinized to an activated clotting time (ACT) greater than 300 seconds.

\section{Operation}

A SENTINEL ${ }^{\mathrm{TM}}$ (Boston Scientific, Malborough, MA) cerebral protection device was placed. The arterial pigtail was positioned at the bottom of the third lumbar vertebral body (L3) as the crossing position. We confirmed the crossing window with aortogram and assessed calcium on the aorta with a rotational cine. From the RCFV, a $55 \mathrm{~cm}$ 7F-IM guiding catheter was positioned at L3. The pigtail catheter was exchanged for a $6 \mathrm{~F} \mathrm{JR}-4.0$ guide catheter via which a $20 \mathrm{~mm}$ Gooseneck snare was advanced and exposed to span the crossing window. Via the IM guide we advanced an 0.014" Astato XS20 wire (Asahi Intecc, Santa Ana, CA), inside a $130 \mathrm{~cm}$ Finecross microcatheter (Terumo, Somerset, NJ), inside a $90 \mathrm{~cm}$ Navicross catheter (Terumo). Biplane fluoroscopy confirmed the crossing position in two orthogonal views. While energizing the wire by electrocautery set to $50 \mathrm{~W}$ cut mode, we traversed from the inferior vena cava (IVC) into the aorta. The wire was snared and advanced to the descending aorta. To facilitate a wire exchange, the Finecross and Navicross were advanced and exchanged for a $0.035^{\prime \prime}$ Lunderquist wire (Cook Medical, Bloomington, IN) advanced through the Navicross. 
The snare and JR-4.0 guide were exchanged for a pigtail catheter, advanced to the aortic root. Over the Lunderquist wire, we exchanged the $8 \mathrm{~F}$ sheath for an $18 \mathrm{~F}$ sheath while visualizing the caval-aortic crossing. Next, a temporary pacemaker was advanced via the LCFV.

We then completed the standard steps of CoreValve implantation using a $29 \mathrm{~mm}$ Evolut Pro+ valve (Medtronic, Dublin, Ireland) using the cusp-overlap technique. The valve was assessed angiographically and with echocardiography, confirming excellent position and no paravalvular leak. The Sentinel device was removed.

Next we closed the caval-aortic tract. Protamine sulfate was administered, and ACT confirmed to be below 180 seconds. A small curved Agilis catheter (Abbott, Abbott Park, IL) was advanced over an $0.032^{\prime \prime}$ wire through the $18 \mathrm{~F}$ sheath along with a $300 \mathrm{~cm} 0.014$ " safety wire to the descending aorta. A 10/8 mm Amplatzer Duct Occluder 1 (ADO1) (St Jude Medical, St. Paul, MN) was advanced through the Agilis. Next, the $18 \mathrm{~F}$ sheath was retrieved into the femoral vein without appreciable change in hemodynamics. The ADO1 was exposed and retrieved with the disk apposed to the aortic wall at the crossing point. After confirming excellent positioning by aortogram, the ADO1 was deployed and the safety wire removed. The $18 \mathrm{~F}$ sheath was removed and site closed with the Perclose devices. A final aortogram 10 minutes later confirmed no extravasation or fistula from the aorta to the IVC (Type 0 closure). All remaining sheaths were removed and the patient was extubated and transferred to the telemetry floor.

\section{Completion}

On post-TAVR day 1 , the patient was hemodynamically stable, in normal sinus rhythm, and ambulating. An echocardiography confirmed no paravalvular leak, AVA $2.10 \mathrm{~cm}^{2}$, and MG $7.0 \mathrm{mmHg}$. He was discharged home with routine 30-day follow-up.

\section{Comments}

\section{Clinical results}

The first series of TC-TAVR was reported in 2014, demonstrating proof of concept and feasibility (1). Following the initial learning curve, a prospective study of 100 TC-TAVR patients reported $99 \%$ successful access, $98 \%$ closure, and inpatient and 30-day survival $96 \%$ and $92 \%$ respectively $(2,3)$. Further, there were no late vascular complications, no deaths related to TC-TAVR access, and a $93 \%$ fistula closure at one year. Survival and heart failure re-admissions were unaffected by fistula patency (3). The survival results were also demonstrated in a series of 491 patients at a single center, with similar Kaplan-Meier survival for TF-TAVR $86 \%$, or TC-TAVR $80 \%$, at 1 year, $\mathrm{P}=0.104$ (4).

At our institution, TC-TAVR is the preferred alternate access for patients with no transfemoral option. We perform over thirty TC-TAVR per year. Anesthesia requirement is determined independently of access strategy. Patients are on the same clinical pathway as minimalist TF-TAVR, with conscious sedation and routine next day discharge.

\section{Advantages}

The major advantage of TC-TAVR is avoidance of violating the thoracic cavity, as both transaortic and transapical TAVR have been shown to have worse outcome (5). From the perspective of the operator, there is considerable ergonomic advantage with TC-TAVR as the entire procedure is completed in the usual TF-TAVR position with appropriate shielding and less radiation exposure. Moreover, there have been well over 700 cases documented in the literature showing that TC-TAVR is easily reproducible and safe.

\section{Caveats}

The caveat to TC-TAVR is the additional time needed for the procedure, however after the initial learning curve, this is less than twenty minutes, ten of which are spent waiting for the final angiogram post aortic closure. The alleged disadvantage of excessive bleeding and need for transfusion seen in the initial series (1) has not been experienced with modern techniques (VARC-2 bleeding reported at $7 \%$ ) (3). For many, this makes the major disadvantage to TC-TAVR the need for specialized equipment, which we do not believe is outside the armamentarium of a modern hybrid interventional laboratory.

\section{Acknowledgments}

Funding: None.

\section{Footnote}

Conflicts of Interest: ABG, VCB, KJG proctor Edwards and 
Medtronic; ABG, VCB equity interest Transmural Systems. The other authors have no conflicts of interest to declare.

Open Access Statement: This is an Open Access article distributed in accordance with the Creative Commons Attribution-NonCommercial-NoDerivs 4.0 International License (CC BY-NC-ND 4.0), which permits the noncommercial replication and distribution of the article with the strict proviso that no changes or edits are made and the original work is properly cited (including links to both the formal publication through the relevant DOI and the license). See: https://creativecommons.org/licenses/by-nc-nd/4.0/.

\section{References}

1. Greenbaum AB, O'Neill WW, Paone G, et al. Caval-aortic access to allow transcatheter aortic valve replacement in otherwise ineligible patients: initial human experience. J Am Coll Cardiol 2014;63:2795-804.

Cite this article as: Byku I, Kamioka N, Greenbaum AB, Gleason P, Babaliaros VC, Grubb KJ. Master of cardiothoracic surgery: transcaval transcatheter aortic valve replacement. Ann Cardiothorac Surg 2020;9(6):531-533. doi: 10.21037/acs-2020av-17
2. Greenbaum AB, Babaliaros VC, Chen MY, et al. Transcaval Access and Closure for Transcatheter Aortic Valve Replacement: A Prospective Investigation. J Am Coll Cardiol 2017;69:511-21.

3. Lederman RJ, Babaliaros VC, Rogers T, et al. The Fate of Transcaval Access Tracts: 12-Month Results of the Prospective NHLBI Transcaval Transcatheter Aortic Valve Replacement Study. JACC Cardiovasc Interv 2019;12:448-56.

4. Paone G, Eng M, Kabbani LS, et al. Transcatheter Aortic Valve Replacement: Comparing Transfemoral, Transcarotid, and Transcaval Access. Ann Thorac Surg 2018;106:1105-12.

5. McCarthy FH, Spragan DD, Savino D, et al. Outcomes, readmissions, and costs in transfemoral and alterative access transcatheter aortic valve replacement in the US Medicare population. J Thorac Cardiovasc Surg 2017;154:1224-32.e1. 